\title{
Preface to the CLEI Electronic Journal Special Issue on Digital Healthcare
}

\author{
Débora Christina Muchaluat Saade \\ Universidade Federal Fluminense, Instituto de Computação, Laboratório MídiaCom \\ Niterói, Brazil, 24210-240 \\ debora@midiacom.uff.br \\ and \\ Jesús Favela \\ Centro de Investigación Científica y de Educación Superior de Ensenada, \\ Departamento de Ciencias de la Computación \\ Baja California, México, 22860 \\ favela@cicese.mx
}

\begin{abstract}
This special issue of the CLEI Electronic Journal (CLEIej) is dedicated to Digital Healthcare. It contains two accepted papers presenting research related to the COVID-19 pandemic in Latin America.
\end{abstract}

Keywords: Digital Healthcare, COVID-19, Machine Learning, Telehealth.

\section{Introduction}

This special issue of the CLEI Electronic Journal (CLEIej) is dedicated to Digital Healthcare. It contains two accepted papers, revised following a single-blind peer-review process. Both papers present research related to the COVID-19 pandemic in Latin America.

The first article, entitled "Using Machine Learning to support health system planning during the COVID-19 pandemic: a case study using data from São José dos Campos (Brazil)", has been developed by Universidade Federal de São Paulo - UNIFESP and Instituto Tecnológico de Aeronáutica - ITA. That work used a dataset with information from people who have undergone the COVID-19 test in the city of São José dos Campos in Brazil. The authors developed predictive models using machine learning techniques to support decision-making related to severity, need for hospitalization and period of hospitalization for patients.

The second paper, entitled "Chatbot as a Telehealth Intervention Strategy in the COVID-19 Pandemic: Lessons Learned from an Action Research Approach", has been developed by Universidade Federal de Minas Gerais in Brazil. That work presents an Action Research approach, which designed a novel chatbot intended to be a first layer of interaction with the public, performing triage of patients and providing information about COVID-19 on a large scale and without human contact.

We would like to thank all the authors that submitted their work to this special issue and all members of the community who were involved in the reviewing process for their anonymous contributions, which ensured the quality of the papers in this issue.

We hope you enjoy the reading and get to know some of the important research about COVID-19 being conducted in Latin America.

Débora Christina Muchaluat Saade - UFF - Brazil

Jesús Favela - CICESE - México 